Pacific Journal of Mathematics

BOUNDS FOR THE SOLUTION OF A CERTAIN CLASS OF
NONLINEAR PARTIAL DIFERENTIAL EQUATIONS

VINOD B. GOYA 


\title{
BOUNDS FOR THE SOLUTIONS OF A CERTAIN CLASS OF NONLINEAR PARTIAL DIFFERENTIAL EQUATIONS
}

\author{
VINOD B. GOYAL
}

This paper is a study of boundedness and other properties of the solutions of nonlinear partial differential equations of the form

$$
\Delta u=P\left(x_{1}, x_{2}, \cdots, x_{n}\right) f(u)
$$

where $P\left(x_{1}, x_{2}, \cdots, x_{n}\right)$ is positive, and $u\left(x_{1}, x_{2}, \cdots x_{n}\right)$ is to be defined in some region of Euclidean $n$-space, and $\Delta u=$ $\sum_{i=1}^{n} \partial^{2} u / \partial x_{i}^{2}$ is the Laplacian of $u$. In particular, we consider the case $f(u)=e^{u}$.

Our principal result is concerned with the nonexistence of entire solutions. An entire solution $u=u\left(x_{1}, x_{2}, \cdots, x_{n}\right)$ will be defined as a solution which though continuous for $0 \leqq r<\infty$ is twice continuously differentiable for $0<r<\infty$. Other results are concerned with the general form of and explicit bounds for solutions.

In the literature on the subject $[3,4,5,8,9,11,12]$ conditions have been given on $f(u)$ in order that the equation

$$
\Delta u=f(u)
$$

or, more generally, the differential inequality

$$
\Delta u \geqq f(u)
$$

will have no solutions $u=u\left(x_{1}, x_{2}, \cdots, x_{n}\right)$ having two continuous derivatives for all finite values of $x_{1}, x_{2}, \cdots, x_{n}$. The most general conditions which exclude such solutions, obtained by Keller [5], are: $f(u)>0, f^{\prime}(u) \geqq 0$ for $-\infty<u<\infty$ and

$$
\int_{0}^{\infty}\left[\int_{0}^{u} f(t) d t\right]^{-1 / 2} d u<\infty
$$

For $n=2$ Redheffer [10] showed that the monotonicity of $f(u)$ may be dispensed with.

In $\S 2$ we shall consider a more general question for the equation

$$
\Delta u=P(x, y) e^{u}, P(x, y)>0, \Delta=\frac{\partial^{2}}{\partial x^{2}}+\frac{\partial^{2}}{\partial y^{2}} \text {. }
$$

While the coefficient $P(x, y)$ will be assumed to be positive and twice continuously differentiable for $0<r<\infty, P(x, y)$ will be permitted to vanish or to become singular in a manner specified in the statement of the Theorem 2.1. If $P(x, y)$ has such a singularity 
it will, of course, be reflected in the singular behaviour of the solutions of (1.4). We shall thus give conditions on $P(x, y)$ which exclude entire solutions of (1.4). An example of such a solution is $u=r$ which solves equation (1.4) with $P(x, y)=e^{-r} / r$.

For $n=2$ it is well known that the function

$$
u(z, \bar{z})=\log \frac{\left|f^{\prime}(z)\right|}{1-|f(z)|^{2}}
$$

is a solution of

$$
\Delta u=4 e^{2 u}
$$

if $f(z)$ is an analytic function satisfying $|f(z)|<1$ and $|f(z)| \neq 0$ in the domain considered. In $\S 3$ we show, conversely, that every solution of (1.6) is essentially of this form. This converse result is necessary if it desired to use (1.5) and the theory of bounded analytic functions to obtain general properties of the regular solutions of (1.6). If the solution $u(z, \bar{z})$ of (1.6) is regular in a disk $|z|<R$, Theorem 3.1 leads to a bound for $u$ in this disk. If $|f(z)|<1$ in $|z|<R$ then, by Schwarz' lemma $\left|f^{\prime}(z)\right| / 1-|f(z)|^{2} \leqq R / R^{2}-|z|^{2}$. Hence, a solution of (1.6) which is regular for $|z|<R$ is subject to the inequality.

$$
u(z, \bar{z}) \leqq \log \frac{R}{R^{2}-|z|^{2}} .
$$

For $z=0$, this leads, in particular, to the well known fact that the equation (1.6) can not have twice continuously differentiable solutions.

In $\S 4$ comparison theorems are proved and explicit bounds are obtained for the solutions of

$$
\Delta u=P(r) f(u)
$$

or, more generally

$$
\Delta u \geqq P(r) f(u) \text {. }
$$

The behaviour of these solutions at an isolated singularity is investigated.

2. Entire solutions. The main result is:

TheOREM 2.1. Let

$$
\iint_{r<r_{0}} P(x, y) d x d y=O\left(r_{0}\right) \quad \text { (for small } r_{0} \text { ) }
$$




$$
\int_{0}^{r} t \sigma(t) d t=O\left(r^{\varepsilon}\right)
$$

where

$$
\sigma(r)=\frac{1}{2 \pi} \int_{0}^{2 \pi} \Delta(\log P) d \theta
$$

If either

$$
\int^{\infty} e^{(1-\beta) g(r)} r^{c-1}(\log r)^{1-3 \beta} d r=\infty
$$

or

$$
\int^{\infty} e^{(1-\beta) g(r)} r^{(1-2 \beta)+\varepsilon^{2}-\varepsilon / 2}(\log r)^{-\beta-\varepsilon} d r=\infty
$$

where

(i ) $c$ is a constant such that $c=(2-\varepsilon)(1-\beta)$ where $1 / 2<\beta<1$ and $\varepsilon>0$ but small. And

(ii) the function $g(r)$ is a solution of

$$
\frac{1}{r} \frac{d}{d r}\left(r \frac{d g}{d r}\right)=\frac{1}{2 \pi} \int_{0}^{2 \pi} \Delta(\log P) d \theta
$$

such that $r g^{\prime}(r) \rightarrow 0$ as $r \rightarrow 0$.

Then (1.4) cannot have a solution which is twice continuously differentiable for $0<r<\infty$ and continuous for $0 \leqq r<\infty$.

That such solutions of (1.4) may exist for certain $P(x, y)$ is shown by the example $u=r^{n}, n \geqq 2$ where $P(x, y)=n^{2} r^{n-2} e^{-r^{n}}$.

Proof. If we set

$$
u=v-\log P
$$

equation (1.4) becomes

$$
\Delta v=e^{v}+\Delta(\log P) .
$$

We introduce the notation

$$
\omega(r)=\frac{1}{2 \pi} \int_{0}^{2 \pi} v(r, \theta) d \theta
$$

By Green's formula for the circle $|z| \leqq r<R$

$$
\iint_{|z| \leqq r} \Delta v d x d y=\int_{|z|=r} \frac{\partial v}{\partial n} d s
$$


where $n$ is the exterior normal. On account of $\partial / \partial n=\partial / \partial r$ it follows that

$$
\int_{0}^{r} \int_{0}^{2 \pi} \Delta v r d \theta d r=\int_{0}^{2 \pi} \frac{\partial v}{\partial r} r d \theta=r \frac{\partial}{\partial r} \int_{0}^{2 \pi} v(r, \theta) d \theta .
$$

With the help of (2.6) and (2.7), this yields

$$
r \frac{d}{d r} \omega(r)=\frac{1}{2 \pi} \int_{0}^{r} \int_{0}^{2 \pi}\left(e^{v}+\Delta(\log P)\right) r d \theta d r .
$$

$\omega(r)$ is single valued and twice continuously differentiable for $r<R$. Because of (2.3) and (2.5), (2.8) is equivalent to

$$
\frac{r d \omega(r)}{d r}=\frac{1}{2 \pi} \int_{0}^{r} \int_{0}^{2 \pi} P(x, y) e^{u} r d \theta d r+\int_{0}^{r} t \sigma(t) d t .
$$

Since $u$ is continuous, it follows from assumption (2.1) and (2.2) that

$$
r \omega^{\prime}(r) \longrightarrow 0
$$

as $r \rightarrow 0$.

Differentiating (2.8) with respect to $r$ and using (2.3), we obtain

$$
\frac{1}{r} \frac{d}{d r}\left(d \frac{d \omega}{d r}\right)=\sigma(r)+\frac{1}{2 \pi} \int_{0}^{2 \pi} e^{v} d \theta .
$$

Since $e^{\xi}$ is convex for all $\xi$, the right hand side of $(2.11)$ can be estimated by

$$
\frac{1}{2 \pi} \int_{0}^{2 \pi} e^{v(r, \theta)} d \theta \geqq e^{1 / 2 \pi} \int_{0}^{2 \pi \int_{0}^{2 \pi} v(r, \theta) d \theta}=e^{\omega(r)} .
$$

Hence (2.11) yields

$$
\frac{d}{d r}\left(r \frac{d \omega}{d r}\right) \geqq r \sigma(r)+r e^{\omega(r)} .
$$

We now set

$$
\omega(r)=g(r)+f(r)
$$

where $g(r)$ is a solution of

$$
\frac{d}{d r}\left(r \frac{d g}{d r}\right)=r \sigma(r)
$$

which is continuous at the origin; that is, we compute $g(r)$ from

$$
r \frac{d}{d r}(g(r))=\int_{0}^{r} t \sigma(t) d t .
$$


Because of our assumption on the behaviour of $\sigma(r)$ at $r=0, g(r)$ will be continuous at $r=0$. Inequality (2.12) then takes the from

$$
\frac{d}{d r}\left(r \frac{d f}{d r}\right) \geqq r \tau(r) e^{f}
$$

where $\tau(r)=e^{g(r)}$. Introducing the new independent variable by $\rho=\log r$ and setting

$$
F=f+2 \rho
$$

inequality (2.15) yields

$$
\ddot{F} \geqq \tau(\rho) e^{F}
$$

where dot denotes the differentiation with respect to $\rho$. Since the right hand side of (2.17) is always positive $F(\rho)$ is convex in $\rho$ therefore, $\omega(r)$ is convex in $\log r$.

Now suppose (1.4) and, therefore, also (2.17) has entire solutions.

We observe that $\dot{F}(\rho)$ must be positive for all $\rho$ in $(-\infty, \infty)$. Indeed, from (2.16), we get, $\dot{F}(\rho)=2+e^{\rho}\left(d f\left(e^{\rho}\right)\right) / d r$. Since by (2.14) and the assumption (2.2), $g^{\prime}(r)=O\left(r^{\varepsilon-1}\right)$ we have, $\lim _{r \rightarrow 0} r g^{\prime}(r)=0$. Hence, by (2.10) and (2.13) $\lim _{r \rightarrow 0} r \omega^{\prime}(r)=\lim _{r \rightarrow 0} r f^{\prime}(r)=0$. It follows, therefore, that $\lim _{\rho \rightarrow-\infty} \dot{F}(\rho)=2$. But, by $(2.17) F(\rho)$ is convex in $\rho$ and we have, consequently,

$$
\dot{F}(\rho) \geqq 2
$$

throughout $(-\infty, \infty)$. It, therefore, follows that $F(\rho)$ is ultimately positive. We choose $\rho_{0}$ large enough so that $F(\rho)>0$ for $\rho>\rho_{0}$ and set

$$
\dot{\phi}=F \dot{F} .
$$

Differentiating with respect to $\rho$ and using (2.17) we have

$$
\dot{\phi} \dot{\phi}^{-r} \geqq \tau F^{1-\gamma} e^{F} \dot{F}^{-\gamma}+F^{-\gamma} \dot{F}^{2-\gamma}
$$

where $\gamma$ is a constant to be chosen later.

Using the inequality [Hardy-Littlewood-Polya] $A+B \geqq(A / \alpha)^{\alpha}(B / \beta)^{\beta}$ where $\alpha+\beta=1,0 \leqq \alpha, \beta \leqq 1$. the inequality (2.20) yields

$$
\dot{\phi} \dot{\phi}^{-\gamma} \geqq \tau^{1-\beta}(1-\beta)^{\beta-1} \beta^{-\beta} e^{(1-\beta) F} F^{1-\beta-\gamma} \dot{F}^{2 \beta-\gamma} .
$$

Now we consider two cases:

Case I. Let $2 \beta-\gamma=0,1 / 2<\beta<1$. Then the inequality (2.21) becomes

$$
\dot{\phi} \phi^{-2 \beta} \geqq C_{1} \tau^{1-\beta} e^{(1-\beta) F} F^{1-3,3}
$$


where $c_{1}=(1-\beta)^{\beta-1} \beta^{-\beta}$. Since $\dot{F} \geqq 2$ we have $F \geqq(2-\varepsilon) \rho$ if $\rho$ is sufficiently large. Moreover, since $e^{(1-\beta) F} F^{(1-3 \beta)}$ is increasing for $F>3 \beta-1 / 1-\beta$, inequality (2.22) yields

$$
\dot{\phi} \phi^{-2 \beta} \geqq c_{2} \tau^{1-\beta} \rho^{1-3 \beta} e^{c \rho}
$$

provided $(2-\varepsilon) \rho>3 \beta-1 / 1-\beta, c_{2}=c_{1}(2-\varepsilon)^{1-3 \beta}$ and $c=(2-\varepsilon)(1-\beta)$. Integration of (2.22) gives

$$
\frac{1}{2 \beta-1}\left[\frac{1}{\phi^{2 \beta-1}\left(\rho_{0}\right)}-\frac{1}{\dot{\phi}^{2 \beta-1}(\rho)}\right] \geqq c_{2} \int_{c \rho_{0}}^{e^{\rho}} e^{(1-\beta) g(r)} r^{c-1}(\log r)^{1-3 \beta} d r
$$

Since $F$ is convex and increasing in $\rho, \phi^{1-2 \beta}(\rho)$ tends to zero as $\rho \rightarrow \infty$. Hence, the left hand side of (2.23) is bounded as $\rho \rightarrow \infty$. This contradicts the assumption (2.4).

Hence the inequality (2.17) and also (1.4) does not have entire solutions.

Case II. Let $2 \beta-\gamma>0,1 / 2<\beta<1$. The inequality (2.21) becomes in this case

$$
\dot{\phi} \dot{\phi}^{-\gamma} \geqq c_{1} \tau^{1-\beta} F^{1-\beta-\gamma} e^{(1-\beta) F} 2^{2 \beta-\gamma}
$$

where we have used (2.18). But since

$$
F^{1-\beta-\gamma} e^{(1-\hat{\beta}) F}>e^{(1-\beta)(2-\varepsilon) \rho}\{(2-\varepsilon) \rho\}^{1-\beta-\gamma}
$$

provided $(2-\varepsilon) \rho>(\gamma+\beta-1)(1-\beta)^{-1}$, we have

$$
\dot{\phi} \phi^{-\gamma} \geqq c_{1} 2^{(2 \beta-\gamma)} \tau^{1-\beta} e^{(1-\beta)(2-\varepsilon) \rho}[\rho(2-\varepsilon)]^{1-\beta-\gamma} .
$$

Choose $\gamma=1+\varepsilon, \varepsilon>0$. Then $\beta>(1+\varepsilon) / 2$. Therefore, integration with respect to $\rho$ gives

$$
\frac{1}{\varepsilon}\left[\frac{1}{\phi^{\varepsilon}\left(\rho_{0}\right)}-\frac{1}{\phi^{\varepsilon}(\rho)}\right] \geqq c_{3} \int^{\rho} e^{(1-\beta) g(r)} r^{(1-2 \beta)+\left(\varepsilon^{2}-\varepsilon / 2\right)}(\log r)^{-\beta-\varepsilon} d r
$$

where $c_{3}=c_{1}(2-\varepsilon)^{-\beta-\varepsilon}$.

If it were true that $u=u(x, y)$ is entire, the left-hand side of (2.24) would remain bounded as $\rho \rightarrow \infty$. Since by $(2.4)^{\prime}$ the right hand side of (2.24) is unbounded, this leads to a contradiction.

This completes the proof of Theorem 2.1.

3. General solution. Let $u(x, y)$ be of class $C^{2}$ in the region $D$ of $x, y$-plane and satisfy (1.6). Introducing the new independent variables $z=x+i y$ and $\bar{z}=x-i y$ equation (1.6) becomes

$$
u_{z \bar{z}}=e^{2 u}
$$


where $\partial / \partial z=1 / 2(\partial / \partial x-i(\partial / \partial y))$ and $\partial / \partial \bar{z}=1 / 2(\partial / \partial x+i(\partial / \partial y))$. How we prove

THEOREM 3.1. Every solution of (1.6) which is twice continuously differentiable in a given region $D$ can be written in the form

$$
u(z, \bar{z})=\log \frac{\left|f^{\prime}(z)\right|}{1-|f(z)|^{2}}
$$

where $f(z)$ is analytic in $D$ such that $\left|f^{\prime}(z)\right| \neq 0$ and $|f(z)|<1$.

Proof. According to an observation which goes back to Bieberbach [1] a regular solution of (1.6) can be associated with an analytic function of $z$ in the following manner: We set

$$
Q=u_{z z}-u_{z}^{2}
$$

where $u$ is a solution of (1.6) or, equivalently, of (3.1) and we compute $Q_{\bar{z}}$. We have, with the help of (3.1), $Q_{\bar{z}}=0$. Thus, $Q$ is found to satisfy the Cauchy-Riemann equations. Since $Q$ is continuous, it must therefore be regular analytic function $\omega(z)$.

If we set

$$
\psi=\bar{e}^{u}
$$

and observe that

$$
\psi_{z z}=\bar{e}^{u}\left(u_{z}^{2}-u_{z z}\right)
$$

we find that $\psi$ is a solution of the linear differential equation

$$
\psi_{z z}+\omega(z) \psi=0 \text {. }
$$

Since $\omega(z)$ is analytic in $z$ the general solution of (3.3) contains the analytic solutions of the equation

$$
F^{\prime \prime}(z)+\omega(z) F(z)=0
$$

because, for an analytic $F$, we have $F^{\prime}(z)=\partial F / \partial z$. The general solution of (3.3) can, therefore, be written in the form

$$
\psi=A^{*} \psi_{1}(z)+B^{*} \psi_{2}(z)
$$

where $\psi_{1}$ and $\psi_{2}$ are two linearly independent (analytic) solutions of $(3.3)^{\prime}$ which may be assumed to be normalized by

$$
\psi_{1} \psi_{2}^{\prime}-\psi_{2} \psi_{1}^{\prime}=1
$$

and $A^{*}$ and $B^{*}$ are constants with respect to $\partial / \partial z$-differentiation used in (3.3) i.e., $\partial A^{*} / \partial z=\partial B^{*} / \partial z=0$. Since these are Cauchy- 
Riemann equations for functions in $\bar{z}$ we have $A^{*}=\overline{A(z)}, B^{*}=\overline{B(z)}$ where $A$ and $B$ are analytic. The general solution of (3.3) is, therefore, found to be of the form

$$
\psi=\overline{A(z)} \psi_{1}(z)+\overline{B(z)} \psi_{2}(z)
$$

where $A, B, \psi_{1}$ and $\psi_{2}$ are analytic functions in $D$. In view of (3.2), equation (3.5) can be written

$$
\bar{e}^{u}=\bar{A}(z) \psi_{1}(z)+\bar{B}(z) \psi_{2}(z) .
$$

Now the proof of the theorem will follow from the following lemma:

LEMMA 3.1. Let $\psi_{1}$ and $\psi_{2}$ be linearly independent solutions of the differential equation (3.3)' where $\omega(z)$ is analytic in $D$. If $A(z)$ and $B(z)$ are analytic in $D$ and if the expression

$$
K(z, \bar{z})=\bar{A}(z) \psi_{1}(z)+\bar{B}(z) \psi_{2}(z)
$$

is real throughout $D$ but does not vanish identically then $K(z, \bar{z})$ can be written in the form

$$
K(z, \bar{z})= \pm|\sigma(z)|^{2} \mp|\tau(z)|^{2}
$$

where $\sigma(z)$ and $\tau(z)$ are two linearly independent solutions of (3.3)' for which

$$
\tau(z) \sigma^{\prime}(z)-\sigma(z) \tau^{\prime}(z)=1
$$

Proof. Since $K(z, \bar{z})$ is real, we have

$$
\bar{A}(z) \psi_{1}(z)+\bar{B}(z) \psi_{2}(z)=A(z) \overline{\psi_{1}(z)}+B(z) \overline{\psi_{2}(z)} .
$$

Differentiation with respect to $z$ and (3.4) give

$$
\bar{\psi}_{1}(z)\left[\psi_{1}^{\prime}(z) A(z)-\psi_{1}(z) A^{\prime}(z)\right]+\bar{\psi}_{2}(z)\left[\psi_{1}^{\prime}(z) B(z)-B^{\prime}(z) \psi_{1}(z)\right]=-\bar{B}(z) .
$$

\section{Setting}

$$
g(z)=\psi_{1}^{\prime}(z) A(z)-\psi_{1}(z) A^{\prime}(z)
$$

and

$$
h(z)=\psi_{1}^{\prime}(z) B(z)-\psi_{1}(z) B^{\prime}(z)
$$

we have

$$
\psi_{1}(z) \bar{g}(z)+\dot{\psi}_{2}(z) \bar{h}(z)=-B(z) .
$$

But the left-hand side of $(3.12)$ is a solution of $(3.3)^{\prime}$; hence $(-B(z))$ satisfies 


$$
B_{z z}+\omega(z) B=0
$$

where $\omega(z)$ is an analytic function. But since $B(z)$ is analytic in $z$,

$$
B^{\prime \prime}(z)+\omega(z) B(z)=0,
$$

consequently, $B$ is of the form

$$
B(z)=\alpha \psi_{1}(z)+\beta \psi_{2}(z)
$$

where $\alpha$ and $\beta$ are constants. Arguing in the same manner (3.4) and (3.9) give

$$
A(z)=\gamma \psi_{1}(z)+\delta \psi_{2}(z)
$$

where $\gamma$ and $\delta$ are constants.

Also from (3.12) and (3.13), $\psi_{1}(z) / \psi_{2}(z)=-\overline{((h(z)+\beta) /(g(z)+\alpha))}$. But since $\psi_{1}(z) / \psi_{2}(z)$ is analytic in $z$ and, moreover, since $\psi_{1}$ and $\psi_{2}$ are linearly independent, we must have $\bar{g}(z)+\bar{\alpha} \equiv 0$ and $\bar{h}(z)+\bar{\beta} \equiv 0$, or equivalently

$$
\left(\gamma \psi_{1}+\delta \psi_{2}\right) \psi_{1}^{\prime}-\left(\gamma \psi_{1}^{\prime}+\delta \psi_{2}^{\prime}\right) \psi_{1}=-\bar{\alpha}
$$

and

$$
\left(\alpha \psi_{1}+\beta \psi_{2}\right) \psi_{1}^{\prime}-\left(\alpha \psi_{1}^{\prime}+\beta \psi_{2}^{\prime}\right) \psi_{1}=-\bar{\beta}
$$

respectively. With the help of (3.12), (3.14), (3.15) and (3.16) the equation (3.7) becomes

$$
K(z, \bar{z})=\gamma\left|\psi_{1}\right|^{2}+\beta\left|\psi_{2}\right|^{2}+\bar{\alpha} \bar{\psi}_{1} \psi_{2}+\alpha \bar{\psi}_{2} \psi_{1} .
$$

Now let $\sigma(z)$ and $\tau(z)$ be any other solutions of (3.3)' such that $\psi_{1}(z)=a \sigma(z)+b \tau(z)$ and $\psi_{2}(z)=c \sigma(z)+d \tau(z)$ where $a, b, c$ and $d$ are constants satisfying

$$
a d-b c=1
$$

and

$$
b(\gamma \bar{a}+\alpha \bar{c})+d(\bar{c} \beta+\bar{a} \bar{\alpha})=0 .
$$

This is possible if the determinant

$$
\boldsymbol{D}=\gamma|a|^{2}+\beta|c|^{2}+2 \operatorname{Re}(\alpha \alpha \bar{c})
$$

does not vanish. Evidently this can always be achieved as long as not all numbers $\alpha, \beta$ and $\gamma$ are zero. However $\alpha, \beta$ and $\gamma$ cannot all be zero since, in view of $(3.17) K(z, \bar{z})$ would then be identically zero, and this case is excluded.

Substituting $\psi_{1}$ and $\psi_{2}$ in (3.17) and using (3.19) we obtain 


$$
\begin{aligned}
K(z, \bar{z})= & |\sigma(z)|^{2}\left\{\gamma|a|^{2}+\beta|c|^{2}+\bar{a} c \bar{\alpha}+a \bar{c} \alpha\right\} \\
& +|\tau(z)|^{2}\left\{\gamma|b|^{2}+\beta|d|^{2}+\bar{b} d \bar{\alpha}+b \bar{d} \alpha\right\} .
\end{aligned}
$$

Now we consider the following two cases:

Case I. Let $\beta \neq 0, \gamma \neq 0$. We set $a \neq 0$ and $c=0$ then, with the help of (3.18) and (3.19), (3.20) becomes

$$
K(z, \bar{z})=|\sigma(z)|^{2} \gamma|a|^{2}+|\tau(z)|^{2}|d|^{2} \gamma^{-1}\left(\beta \gamma-|\alpha|^{2}\right) .
$$

(i) Let $\gamma>0, \beta \gamma-|\alpha|^{2}=m$ ( $m$ is a positive integer). Hence,

$$
K(z, \bar{z})=\left|\sigma^{*}\right|^{2}+\left|\tau^{*}\right|^{2}
$$

where $\sigma^{*}=\sigma\left(\gamma|a|^{2}\right)^{1 / 2}$ and $\tau^{*}=\tau m^{1 / 2}\left(\gamma|a|^{2}\right)^{-1 / 2}$ are solutions of (3.3)'.

(ii) $\gamma>0, \beta \gamma-|\alpha|^{2}=-m$. In this case

$$
K(z, \bar{z})=\left|\sigma^{*}\right|^{2}-\left|\tau^{*}\right|^{2} \text {. }
$$

(iii) Let $\gamma<0, \beta \gamma-|\alpha|^{2}=m$. Then

$$
K(z, \bar{z})=-\left|\sigma^{*}\right|^{2}-\left|\tau^{*}\right|^{2}
$$

(iv) $\gamma<0, \beta \gamma-|\alpha|^{2}=-m$. This gives

$$
K(z, \bar{z})=-\left|\sigma^{*}\right|^{2}+\left|\tau^{*}\right|^{2} \text {. }
$$

Case II. Let $\beta=0, \gamma=0$. We set $a, b \neq 0$. With this choice (3.18) and (3.19) reduce (3.20) to

$$
K(z, \bar{z})=-\left|\sigma_{1}\right|^{2}+\left|\tau_{1}\right|^{2}
$$

where $\left|\sigma_{1}\right|=|\sigma|(\bar{a} \bar{\alpha})^{1 / 2} b^{-1 / 2}$ and $\left|\tau_{1}\right|=a^{-1 / 2}|\tau|(\bar{\alpha} \bar{\beta})^{1 / 2}$ and are solutions of $(3.3)^{\prime}$.

Summing up, we have thus proved that, if the function $K(z, \bar{z})$ is real, it must have either of the three following forms

$$
\left.\begin{array}{l}
K(z, \bar{z})=|\tau|^{2}-|\sigma|^{2} \\
K(z, \bar{z})=|\tau|^{2}+|\sigma|^{2} \\
K(z, \bar{z})=-|\tau|^{2}-|\sigma|^{2}
\end{array}\right\}
$$

where $\sigma$ and $\tau$ are solutions of the differential equation (3.3)' normalized by (3.8). The case $K(z, \bar{z})=|\sigma|^{2}-|\tau|^{2}$ is evidently not essentially different from case (1). Case (3) can be excluded immediately, since beacuse of (3.6) and (3.7) $K(z, \bar{z})$ must be positive. This also shows that, in case (1), we necessarily must have

$$
|\tau(z)|>|\sigma(z)| \text {. }
$$

We now define 


$$
f(z)=\frac{\sigma(z)}{\tau(z)}
$$

In view of (3.8) we have

$$
f^{\prime}(z)=\frac{1}{\tau^{2}(z)}
$$

and thus $|\sigma|^{2}+|\tau|^{2}=\left(1+|f(z)|^{2}\right) /\left|f^{\prime}(z)\right|$ in case (2) and $|\tau|^{2}-|\sigma|^{2}=$ $\left(1-|f(z)|^{2}\right) /\left|f^{\prime}(z)\right|$ in case (1). Comparing this with (3.6), (3.7) and $(S)$ we find that $u(z, \bar{z})$ must be either of the forms

$$
\begin{aligned}
& u(z, \bar{z})=\log \frac{\left|f^{\prime}(z)\right|}{1-|f(z)|^{2}} \\
& u(z, \bar{z})=\log \frac{\left|f^{\prime}(z)\right|}{1+|f(z)|^{2}} \\
& u(z, \bar{z})=\log \frac{1+|f(z)|^{2}}{\left|f^{\prime}(z)\right|} .
\end{aligned}
$$

Since the last two functions are not solutions of (1.6), these cases are excluded. Hence any real solution of (1.6) must be of the from

$$
u(z, \bar{z})=\log \frac{\left|f^{\prime}(z)\right|}{1-|f(z)|^{2}}
$$

where because of (3.21) and (3.22) $|f(z)|<1$ and in view of (3.23) $\left|f^{\prime}(z)\right| \neq 0$.

This completes the proof of Theorem 3.1.

4. Bounds for the solutions of $\Delta_{u} \geqq P(r) f(u)$. Let

$$
\Delta=\frac{\partial^{2}}{\partial x_{1}^{2}}+\frac{\partial^{2}}{\partial x_{2}^{2}}+\cdots+\frac{\partial^{2}}{\partial x_{n}^{2}}
$$

denote the $n$-dimensional Laplace operator and let $D_{r}$ and $S_{r}$ stand for the open sphere $x_{1}^{2}+x_{2}^{2}+\cdots+x_{n}^{2}<r^{2}$ and its boundary

$$
x_{1}^{2}+x_{2}^{2}+\cdots+x_{n}^{2}=r^{2}
$$

respectively. We are concerned here with functions

$$
\omega=\omega(Q)\left(Q \in D_{r}, 0<r<R\right)
$$

which are of class $C^{2}$ in $D_{r}$ and satisfy the differential equation

$$
\Delta \omega=P(r) F(\omega)
$$

or, more generally, the differential inequality

$$
\Delta \omega \geqq P(r) F(\omega) .
$$


Nehari [6] found explicit bounds for the solutions of the differential equation $\Delta u=F(u)$ or, more generally the differential inequality $\Delta u \geqq F(u)$ which are regular in a disk. We shall obtain here a more general result, which also applies to certain equations of the form (4.1).

LEMMA 4.1. Let $F(t)$ and $G(t)$ be positive and differentiable functions for $-\infty<t<\infty$ and such that the integrals

$$
\int_{\omega}^{\infty} \frac{d t}{F(t)}, \int_{v}^{\infty} \frac{d t}{G(t)}
$$

exist, and let $\omega=\omega\left(x_{1}, x_{2}, \cdots, x_{n}\right)$ and $v=v\left(x_{1}, x_{2}, \cdots x_{n}\right)$ be two functions related by the identity

$$
\int_{\omega}^{\infty} \frac{d t}{F(t)}=\int_{v}^{\infty} \frac{d t}{G(t)}
$$

Then

$$
\frac{\Delta \omega}{F(\omega)} \geqq \frac{\Delta v}{G(v)}
$$

provided $F^{\prime}(\omega) \geqq G^{\prime}(v)$.

Proof. We write $x$ for one of the variables $x_{1}, x_{2}, \cdots, x_{n}$ and differentiate (4.2) twice with respect to $x$. This yields

$$
\begin{aligned}
& -\frac{v_{x}}{G(v)}=-\frac{\omega_{x}}{F(\omega)} \\
& -\frac{v_{x x}}{G(v)}+\frac{v_{x}^{2} G^{\prime}(v)}{G^{2}(v)}=-\frac{\omega_{x x}}{F(\omega)}+\frac{v_{x}^{2} F^{\prime}(\omega)}{G^{2}(v)} .
\end{aligned}
$$

Summing over all $x_{n}$ and using the fact that $F^{\prime}(\omega) \geqq G^{\prime}(v)$, we get (4.3).

We derive the following corollary.

COROLlaRY 5.1. If $v=v\left(x_{1}, x_{2}, \cdots, x_{n}\right)$ is a function satisfying the differential inequality

$$
\Delta v \leqq P v^{k}, \quad k>1
$$

where $P=P\left(x_{1}, x_{2}, \cdots, x_{n}\right)$ is positive, and if $F(u)$ is such that

$$
F^{\prime}(u) \int_{u}^{\infty} \frac{d t}{F(t)} \leqq \frac{k}{k-1}
$$

then, the function $u$ defined by

$$
\frac{1}{(k-1) v^{k-1}}=\int_{u}^{\infty} \frac{d t}{F(t)}
$$


is subject to the inequality

$$
\Delta u \leqq P F(u) .
$$

Setting $G(v)=v^{k}$ in Lemma 4.1, the proof of the Corollary 4.1 is immediate.

As an application of Corollary 4.1, we prove the following result.

THEOREM 4.1. If the function $\omega=\omega\left(x_{1}, x_{2}, \cdots, x_{n}\right)$ satisfies the inequality

$$
\Delta \omega \geqq r^{2} F(\omega)
$$

where $F(\omega)$ is such that $F^{\prime}(\omega) \int^{\infty} d t / F(t) \leqq 9 / 8$ and $F^{\prime}(\omega) \geqq 0$ then the function $u$ defined by

$$
\frac{\left(r^{2}-\rho^{2}\right)^{2}\left(R^{2}-r^{2}\right)^{2}}{20 R^{4}}=\int_{u}^{\infty} \frac{d t}{F(t)} \quad 0<\rho<r<R
$$

is such that

$$
\omega \leqq u
$$

Proof. Consider the function $v$ defined by

$$
v=\frac{1}{\left(r^{2}-\rho^{2}\right)^{\alpha}\left(R^{2}-r^{2}\right)^{\alpha}}
$$

where $\alpha$ is a constant to be determined later. Differentiating (4.9) twice with respect to one of the variables $x=x_{k}$, we obtain

$$
\begin{aligned}
v_{x}= & -\frac{2 x \alpha}{\left(r^{2}-\rho^{2}\right)^{\alpha+1}\left(R^{2}-r^{2}\right)^{\alpha}}+\frac{2 x \alpha}{\left(r^{2}-\rho^{2}\right)^{\alpha}\left(R^{2}-r^{2}\right)^{\alpha+1}} \\
v_{x x}= & -\frac{2 \alpha}{\left(R^{2}-r^{2}\right)^{\alpha}\left(r^{2}-\rho^{2}\right)^{\alpha+1}}+\frac{4 x^{2} \alpha(\alpha+1)}{\left(R^{2}-r^{2}\right)^{\alpha}\left(r^{2}-\rho^{2}\right)^{\alpha+2}} \\
& +\frac{2 \alpha}{\left(r^{2}-\rho^{2}\right)^{\alpha}\left(R^{2}-r^{2}\right)^{\alpha+1}}-\frac{8 x^{2} \alpha^{2}}{\left(r^{2}-\rho^{2}\right)^{\alpha+1}\left(R^{2}-r^{2}\right)^{\alpha+1}} \\
& +\frac{4 x^{2} \alpha(\alpha+1)}{\left(r^{2}-\rho^{2}\right)^{\alpha}\left(R^{2}-r^{2}\right)^{\alpha+2}} \cdot
\end{aligned}
$$

Summing over all $x=x_{k}$ and choosing $\alpha \geqq 1 / 4$ we get,

$$
\Delta v \leqq \frac{5}{2} r^{2} R^{4} v^{9}
$$

Now let $v=\left(2^{1 / 8} y\right) /\left(5^{1 / 2} R^{2}\right)^{1 / 4}$ then we have

$$
\Delta y \leqq r^{2} y^{9}
$$


where $y$ is given by

$$
y=\left(\frac{R^{2} 5^{1 / 2} 2^{-1 / 2}}{\left(R^{2}-r^{2}\right)\left(r^{2}-\rho^{2}\right)}\right)^{1 / 4} .
$$

Now applying Corollary 4.1 to (4.10), we obtain,

$$
\Delta u \leqq r^{2} F(u)
$$

when $u$ is defined by

$$
\frac{\left(r^{2}-\rho^{2}\right)^{2}\left(R^{2}-r^{2}\right)^{2}}{20 R^{4}}=\int_{u}^{\infty} \frac{d t}{F(t)} .
$$

Clearly, $u^{\prime}(0)=0$ and $u \rightarrow \infty$ as $r \rightarrow R$ or $\rho \rightarrow r$. The fact that $\omega \leqq u$ now follows from Osserman's lemma [8]. This proves our assertion.

THEOREM 4.2. Let $f(\omega)$ be positive, nondecreasing, differentiable function in $(-\infty, \infty)$ for which

$$
\int_{\omega}^{\infty} \frac{d t}{f(t)}
$$

exists and

$$
f^{\prime}(\omega) \int_{\omega}^{\infty} \frac{d t}{f(t)} \leqq 1+\lambda
$$

If

$$
u(r)=\sup _{Q \in S_{r}} \omega(Q)
$$

where $\omega(Q)$ ranges over all functions of class $C^{2}$ in $D_{r}$ which satisfy (4.1). Then

$$
\frac{C(\lambda) \alpha\left(R^{2}-r^{2}\right)^{2}}{R^{2}} \leqq \int_{u(r)}^{\infty} \frac{d t}{f(t)}
$$

in case $P(r)=\alpha(\alpha>0)$.

$$
\frac{C(\lambda) \beta r^{n / 1+\lambda}\left(R^{2}-r^{2}\right)^{2}}{R^{2}} \leqq \int_{u(r)}^{\infty} \frac{d t}{f(t)}
$$

if $P(r)=\beta r^{n / 1+\lambda}(\beta>0)$ and

$$
\frac{C(\lambda) \gamma r^{n-2 / \lambda}\left(R^{2}-r^{2}\right)^{2}}{R^{2}} \leqq \int_{u(r)}^{\infty} \frac{d t}{f(t)}
$$

if $P(r)=\gamma r^{n-2 / \lambda}(\gamma>0)$ 
where

$$
C(\lambda)=\frac{1}{4 n}
$$

and

$$
C(\lambda)=\frac{1}{8(2 \lambda+1)}
$$

The inequalities (4.12), (4.13) and (4.14) are sharp.

The case $\lambda=0$ had been considered by the author in [2].

Proof. Consider the function $g=g(r)$ defined by

$$
\frac{C(\lambda)\left(R^{2}-r^{2}\right)^{2}}{R^{2}}=\frac{1}{p(r)} \int_{g}^{\infty} \frac{d t}{f(t)}
$$

where $p(r)$ is positive, monotonically increasing and twice continuously differentiable and $C$ is a positive constant to be chosen later. Denoting by $x$ one of the variables $x_{k}$ and differentiating twice with respect to $x$ we have

$$
\begin{aligned}
-\frac{4 c x\left(R^{2}-r^{2}\right)}{R^{2}}=- & \frac{g_{x}}{p(r) f(g)}-\frac{2 x}{p^{2}(r)} \int_{g}^{\infty} \frac{d t}{f(t)} \\
-\frac{4 c\left(R^{2}-r^{2}\right)}{R^{2}}=- & \frac{8 c x^{2}}{R^{2}}-\frac{g_{x x}}{p(r) f(g)}+\frac{4 x \dot{p}(r) g_{x}}{p^{2}(r) f(g)}+\frac{g_{n}^{2} f^{\prime}(g)}{p(r) f^{2}(g)} \\
& \frac{2 \dot{p}(r)}{p^{2}(r)} \int_{g}^{\infty} \frac{d t}{f(t)}-\frac{4 x^{2} \ddot{p}(r)}{p^{2}(r)} \int_{g}^{\infty} \frac{d t}{f(t)} \\
& \frac{8 x^{2} \dot{p}^{2}(r)}{p^{3}(r)} \int_{g}^{\infty} \frac{d t}{f(t)}
\end{aligned}
$$

where dot denotes differentiation with respect to $r^{2}$. With the help of (4.17) and (4.18), (4.19) becomes

$$
\begin{aligned}
\frac{g_{x x}}{p(r) f(g)}= & -\frac{8 c x^{2}}{R^{2}}+\frac{4 c\left(R^{2}-r^{2}\right)}{R^{2}}+\frac{16 c x^{2} \dot{p}(r)\left(R^{2}-r^{2}\right)}{R^{2} p(r)}+\frac{4 c x^{2}}{R^{2}} \\
& \times f^{\prime}(g) c p(r) \frac{\left(R^{2}-r^{2}\right)^{2}}{R^{2}}\left[2-\frac{\dot{p}(r)\left(R^{2}-r\right)^{2}}{p(r)}\right]^{2} \\
& -\frac{2}{p^{2}(r)}\left(2 x^{2} \ddot{p}(r)+\dot{p}(r)\right) \int_{g}^{\infty} \frac{d t}{f(t)}
\end{aligned}
$$

Summing over all $x_{k}$ and using (4.11) it reduces to 


$$
\begin{aligned}
\frac{\Delta g}{p(r) f(g)} \leqq & 4 c\left\{n-\frac{r^{2}}{R^{2}}(n-2-4 \lambda)\right\}-\frac{16\left(R^{2}-r^{2}\right) c r^{2} \dot{p}(r)}{R^{2}} \lambda \\
& -\frac{2 c\left(R^{2}-r^{2}\right)^{2}}{R^{2}}\left\{\frac{2 r^{2} \ddot{p}(r)+n \dot{p}(r)}{p(r)}-\frac{2 r^{2} \dot{p}^{2}(r)}{p^{2}(r)}(1+\lambda)\right\}
\end{aligned}
$$

We now consider the following cases:

Case I. Choose $p(r)$ such that $\dot{p}(r) / p(r)\left(\left(2 r^{2} \dot{p}(r) / p(r)\right)-n /(1+\lambda)\right)=0$.

(i) If $\dot{p}=0$ or $p=\alpha$ where $\alpha$ is an arbitrary positive constant then (4.20) becomes

$$
\frac{\Delta g}{\alpha f(g)} \leqq 4 c\left\{n-\frac{r^{2}}{R^{2}}(n-2-4 \lambda)\right\} .
$$

If, $4 \lambda \leqq n-2$ it follows that $\Delta g \leqq 4 n c \alpha f(g)$ and if $C$ is given by (4.15), we have

$$
\Delta g \leqq \alpha f(g) \text {. }
$$

If $4 \lambda>n-2$ the right hand of (4.21) attains maximum for $R=r$ and the value of (4.16) for $C$ again leads to (4.22). Since $\dot{g}(0)=0$ and increases to $\infty$ as $r \rightarrow R$ the proof of (4.12) will follow from Osserman's lemma [8].

REMARK. If $\alpha=1$ the left hand inequality (9) of Theorem 1 of Nehari [6] becomes a particular case of this result.

(ii) If $2 r^{2} \dot{p}(r) / p(r)-(n / 1+\lambda)=0$ or $p=r^{n / 1+\lambda} \beta$ where $\beta$ is an arbitrary positive constant then (4.20) gives

$$
\frac{\Delta g}{\beta r^{n / 1+\lambda} f(g)} \leqq 4 c\left\{n-\frac{r^{2}}{R^{2}}(n-2-4 \lambda)\right\} \text {. }
$$

If $C$ is given by the values (4.15) and (4.16), we have

$$
\Delta g \leqq \beta r^{n / 1+\lambda} f(g) \text {. }
$$

Now the proof of (4.13) will follow from Osserman's lemma [8].

Case II. Assume $p(r)$ to satisfy

$$
2 r^{2} p(r)(\ddot{p} r)+n p(r) \dot{p}(r)-2 r^{2}(1+\lambda) \dot{p}^{2}(r)=0
$$

or $p(r)=\gamma r^{n-2 / \lambda}$ where $\gamma$ is an arbitrary positive constant. Then (4.20) reduces to

$$
\frac{\Delta g}{\gamma r^{n-2 / 2} f(g)} \leqq 4 c\left\{n-\frac{r^{2}}{R^{2}}(n-2-4 \lambda)\right\} .
$$

Now if $C$ takes the values (4.15) and (4.16) respectively, we have 


$$
\Delta g \leqq \gamma r^{n-2 / \lambda} f(g)
$$

and (4.14) is proved with the help of Osserman's lemma [8]. We derive the following corollary:

COROLlary 4.2. If $\omega$ satisfies the equation

$$
\Delta \omega=\beta r^{n / 1+\lambda} \omega^{1+(1 / \lambda)} \quad(\lambda>0, n \geqq 2)
$$

where $\beta$ is an arbitrary constant, then

$$
\omega \leqq\left(\frac{\lambda R^{2}}{c(\lambda) \beta r^{n / 1+\lambda}\left(R^{2}-r^{2}\right)^{2}}\right)^{\lambda} .
$$

Also the behaviour of $\omega$ is such that

$$
\varlimsup_{r \rightarrow 0}\left(\frac{\log \omega}{\log 1 / r}\right) \leqq \frac{n \lambda}{1+\lambda} .
$$

Indeed, setting $f(t)=t^{1+(1 / \lambda)}$ in (4.13), we have (4.23), where $\omega=u$. Taking logarithm on both sides, we have, from (4.23)

$$
\log \omega \leqq \lambda \log \frac{\lambda R^{2}}{\beta c(\lambda)\left(R^{2}-r^{2}\right)^{2}}+\frac{n \lambda}{1+\lambda} \log \frac{1}{r} .
$$

Dividing by $\log 1 / r$ and letting $r \rightarrow 0$

$$
\varlimsup_{r \rightarrow 0}\left(\frac{\log \omega}{\log 1 / r}\right) \leqq \frac{n \lambda}{1+\lambda} .
$$

A similar result could also be proved about the solutions of the equation

$$
\Delta \omega=\gamma r^{n-2 / \lambda} \omega^{1+(1 / \lambda)} .
$$

The next theorem concerns the lower bounds for the maximum of the solutions of (4.1).

THEOREM 4.3. Let $f(\omega)$ satisfy the conditions of theorem 4.2 with (4.11) replaced by

$$
f^{\prime}(\omega) \int_{\omega}^{\infty} \frac{d t}{f(t)}=1+\lambda
$$

If

$(\mathrm{G})^{\prime}$

$$
v(r)=\operatorname{Sup}_{Q \in S_{r}} \omega(Q)
$$

where $\omega(Q)$ ranges over all functions of class $C^{2}$ in $D_{r}$ and which satisfy (4.1) then 


$$
\int_{v}^{\infty} \frac{d t}{f(t)} \leqq \frac{\kappa\left(R^{2}-r^{2}\right)}{2 n}
$$

if $p(r)=\kappa$ where $\kappa$ is an arbitrary positive constant,

$$
\int_{v}^{\infty} \frac{d t}{f(t)} \leqq \frac{\delta r^{n-2 / \lambda-1}\left(R^{2}-r^{2}\right)}{2 n} \quad\left(n>2, \lambda>1, n<\frac{4 \lambda}{1+\lambda}\right)
$$

provided $p(r)=\delta r^{n-2 / \lambda-1}(\delta>0)$.

$$
\int_{v}^{\infty} \frac{d t}{f(t)} \leqq \frac{\mu r^{1 / \lambda}\left(R^{2}-r^{2}\right)}{6}
$$

in case $p(r)=\mu r^{1 / 2}(\mu>0)$. However, in 2-dimensional case

$$
\int_{v}^{\infty} \frac{d t}{f(t)} \leqq \frac{\nu r^{l}\left(R^{2}-r^{2}\right)}{4}
$$

where $p(r)=\nu r^{l}$, $\nu$ and $l$ being arbitrary positive constants.

Proof. Consider the function $h=h(r)$ defined by

$$
\frac{\rho^{2}-r^{2}}{2 n}=\frac{1}{p(r)} \int_{h}^{\infty} \frac{d t}{f(t)}
$$

where $p(r)$ is positive, monotonically increasing and twice continuously differentiable. Clearly, $h$ belongs to the class $C^{2}$ in $D_{r}$. Differentiating (5.28) twice with respect to $x=x_{k}$ we obtain

$$
\begin{aligned}
-\frac{n}{x}= & -\frac{h_{x}}{f(h) p(r)}-\frac{2 x \dot{p}(r)}{p^{2}(r)} \int_{h}^{\infty} \frac{d t}{f(t)} \\
-\frac{1}{n}= & -\frac{h_{x x}}{f(h) p(r)}+\frac{4 x h_{x} \dot{p}(r)}{f(h) p^{2}(r)}+\frac{h_{x}^{2} f^{\prime}(h)}{p(r) f^{2}(h)}-\frac{2 \dot{p}}{p^{2}} \int_{h}^{\infty} \frac{d t}{f(t)} \\
& -\frac{4 x^{2} \ddot{p}(r)}{p^{2}(r)} \int_{h}^{\infty} \frac{d t}{f(t)}+\frac{8 x^{2} \dot{p}(r)}{p^{3}(r)} \int_{h}^{\infty} \frac{d t}{f(t)} .
\end{aligned}
$$

Using (4.29) and summing over all $x_{k}$, we obtain

$$
\begin{aligned}
\frac{\Delta h}{f(h) p(r)}=1 & +\frac{4 r^{2} \dot{p}(r)}{n p(r)}+r^{2} p(r) f^{\prime}(h)\left[\frac{\rho^{2}-r^{2}}{n} \frac{\dot{p}}{\rho}-\frac{1}{n}\right]^{2} \\
& -\frac{2 r^{2} \ddot{p}+n \dot{p}}{p} \times \frac{\rho^{2}-r^{2}}{n} .
\end{aligned}
$$

Since $f^{\prime}>0$ we obtain with the help of $(4.11)^{\prime}$

$$
\frac{\Delta h}{f(h) p(r)} \geqq 1-\frac{4 r^{2} \dot{p}}{n p} \lambda-\frac{\rho^{2}-r^{2}}{n}\left[\frac{n \dot{p}+2 r^{2} \ddot{p}}{p}-(1+\lambda) \frac{2 r^{2} \dot{p}_{\dot{p}}^{2}}{p^{2}}\right] .
$$

Now we consider the following cases. 
Case I. Choose $p$ such that $\dot{p}=0$ or, $p=\kappa$ where $\kappa$ is an arbitrary positive constant. Hence (4.30) reduces to

$$
\Delta h \geqq \kappa f(h) \text {. }
$$

Consequently $(G)^{\prime}$ implies

$$
h(r) \leqq v(r) \text {. }
$$

Since we can take $\rho$ arbitrarily close to $R$, we have

$$
\int_{v}^{\infty} \frac{d t}{f(t)} \leqq \frac{\kappa\left(R^{2}-r^{2}\right)}{2 n}
$$

Case II. Assume $p(r)$ to be such that

$$
n \dot{p}(r) p(r)+2 r^{2} p(r) \ddot{p}(r)-2 \lambda r^{2} \dot{p}^{2}(r)=0
$$

or $p=\delta r^{n-2 / \lambda-1}$ where $\delta$ is an arbitrary positive constant, $n>2$, $\lambda>1$ and such that $n<(4 \lambda / 1+\lambda)$. Hence (4.30) becomes

$$
\Delta h \geqq\left\{1-\frac{2 \lambda(n-2)}{n(\lambda-1)}\right\} \delta r^{n-2 / \lambda-1} f(h) .
$$

Using $(G)^{\prime}$ and arguing as above, we obtain

$$
\int_{v}^{\infty} \frac{d t}{f(t)} \leqq \frac{\delta r^{n-2 / 2-1}\left(R^{2}-r^{2}\right)}{2 n}
$$

Case III. Choose $p$ to satisfy

$$
n p(r) \dot{p}(r)+2 r^{2} p(r) \ddot{p}(r)-(1+\lambda) 2 r^{2} \dot{p}^{2}(r)=0
$$

or $p=\mu r^{1 / \lambda}$ where $\mu$ is an arbitrary positive constant and $n=3$. Hence (4.30) gives

$$
\Delta h \geqq \frac{\mu}{3} r^{1 / \lambda} f(h)
$$

Using the same argument as above, we have

$$
\int_{v}^{\infty} \frac{d t}{f(t)} \leqq \frac{\mu r^{1 / \lambda}\left(R^{2}-r^{2}\right)}{6}
$$

Case IV. Assume $p$ to be such that $2 r^{2} p \ddot{p}+n p \dot{p}-2 r^{2} \dot{p}^{2}=0$ or $p=\nu r^{l}$ where $\nu$ and $l$ are arbitrary positive constants. Consequently

$$
\Delta h \geqq \nu(1-l \lambda) r^{l} f(h) \text {. }
$$

And, as above we conclude 


$$
\int_{v}^{\infty} \frac{d t}{f(t)} \leqq \frac{\nu r^{l}\left(R^{2}-r^{2}\right)}{4}
$$

This completes the proof of the theorem.

We derive the following corollaries:

CoROLlaRy 4.3. In case of a function $\omega$ regular in $D_{r}$ and which satisfies the differential equation

$$
\Delta \omega=\delta r^{n-2 / \lambda-1}\left\{1-\frac{2 \lambda(n-2)}{n(\lambda+1)}\right\} \omega^{1+(1 / \lambda)}
$$

where $\delta$ is an arbitrary positive constant, $n>2, \lambda>1$ and such that $n<(4 \lambda / 1+\lambda)$ we have

$$
\left(\frac{2 n \lambda}{\delta r^{n-2 / \lambda-1}\left(R^{2}-r^{2}\right)}\right)^{\lambda} \leqq \omega
$$

And also the behaviour of $\omega$ is such that

$$
\varlimsup_{r \rightarrow 0}\left(\frac{\log \omega}{\log 1 / r}\right) \geqq \lambda \frac{n-2}{\lambda-1} .
$$

Indeed, setting $f(t)=t^{1+(1 / \lambda)}$ in (4.25), where $v=\omega$, we obtain

$$
\omega^{1 / \lambda} \geqq \frac{2 n \lambda}{\delta r^{n-2 / \lambda-1}\left(R^{2}-r^{2}\right)} .
$$

Taking logarithm on both sides, we get

$$
\log \omega \geqq \lambda \log \frac{2 n \lambda}{\delta\left(R^{2}-r^{2}\right)}+\lambda \frac{n-2}{\lambda-1} \log \frac{1}{r} .
$$

Dividing by $\log 1 / r$ and taking the limit

$$
\varlimsup_{r \rightarrow 0}\left(\frac{\log \omega}{\log 1 / r}\right) \geqq \lambda \frac{n-2}{\lambda-1} .
$$

COROLLARY 4.4. If $\Delta=\partial^{2} / \partial x_{1}^{2}+\partial^{2} / \partial x_{2}^{2}+\partial^{2} / \partial x_{3}^{2}$ is a 3-dimensional Laplace operator and $\omega$ satisfies the equation

$$
\Delta \omega=\frac{\mu}{3} r^{1 / \lambda} \omega^{1+(1 / \lambda)}
$$

we have

$$
\omega \geqq\left(\frac{6}{\mu r^{1 / \lambda}\left(R^{2}-r^{2}\right)}\right)^{\lambda}
$$


and

$$
\varlimsup_{r \rightarrow 0}\left(\frac{\log \omega}{\log 1 / r}\right) \geqq 1
$$

COROLLARY 4.5. If the function $\omega$ is regular in $D_{r}$ and satisfies the differential equation

$$
\Delta \omega=\delta(1-l \lambda) r^{l} \omega^{1+(1 / \lambda)} \quad\left(\Delta=\frac{\partial^{2}}{\partial x_{1}^{2}}+\frac{\partial^{2}}{\partial x_{2}^{2}}\right)
$$

we have

$$
\left(\frac{4}{\delta r^{l}\left(R^{2}-r^{2}\right)}\right)^{2} \leqq \omega
$$

and also the behaviour of $\omega$ is such that

$$
\varlimsup_{r \rightarrow 0}\left(\frac{\log \omega}{\log 1 / r}\right) \geqq l \lambda .
$$

The proof of Corollaries 4.4 and 4.5 is exactly the same as that of 4.3 .

\section{REFERENCES}

1. L. Bieberbach, $\Delta u=e^{u}$ und die automorphen Funktionen, Math. Ann. 77 (1916), 173-212.

2. V. B. Goyal, Bounds for the solutions of $\Delta u \geqq p(r) f(u)$, Compositio Mathematica 18 (1967), 162-169.

3. E. K. Haviland, A note on unrestricted solutions of the differential equation $\Delta u=$ $f(u)$, J. London Math. Soc. 26 (1951), 210-214.

4. E. Hopf, On non-linear partial differential equations, Berkeley Symposium on Partial Differential Equations (Lecture series), 1957, 1-31.

5. J. D. Keller, On solutions of $\Delta u=f(u)$, Comm. Pure Appl. Math. 10 (1957), 503510.

6. Z. Nehari, Bounds for the solutions of a class of non-linear partial differential equations, Oroc. Math. Soc. (1963), 829-836.

7. J. Nitsche, Uber die isolierten Singularitäten der Lösungen von $\Delta u=e^{u}$, Math. Zeit. 68 (1957-58), 316-324.

8. R. Osserman, On the inequality $\Delta u \geqq f(u)$, Pacific J. Math 7 (1957), 1641-1647.

9. R. Redheffer, On entire solutions of non-linear equations (abstract), Bull. Amer. Math. Soc. 62 (1956), 408.

10. - On the inequality $\Delta u \geqq f(u,|\operatorname{grad} u|)$, J. Math. Anal. Appl. 1 (1960), 277-299.

11. W. Walter, Überganze Lösungen der Differentialgleichung $\Delta u=f(u)$, Jahresb. d. Deutsch. Math. Ver. 57 (1955), 94-102.

12. H. Wittich, Ganze Lösungen der Differentialgleichung $\Delta u=e^{u}$, Math. Zeit. 49 (1943-44), 579-582.

Received January 20, 1966, in revised form January 27, 1967, and July 22, 1969. 
This work is a portion of the author's doctoral dissertation written under the direction of Professor Z. Nehari at Carnegie Institute of Technology in 1963, and was supported by the United States Air Force under contract No. AF20A.

KURUKSHETRA UNIVERSITY

KURUKSHETRA, HARYANA, INDIA 


\section{PACIFIC JOURNAL OF MATHEMATICS}

\section{EDITORS}

H. SAMELSON

Stanford University

Stanford, California 94305

\section{RichaRd PIERCe}

University of Washington

Seattle, Washington 98105
J. DUGUNDJI

Department of Mathematics

University of Southern California

Los Angeles, California 90007

RICHARD ARENS

University of California

Los Angeles, California 90024

\section{ASSOCIATE EDITORS}

E. F. BECKENBACH

B. H. NeUManN

F. WOLF

K. YosHIDA

\section{SUPPORTING INSTITUTIONS}

UNIVERSITY OF BRITISH COLUMBIA CALIFORNIA INSTITUTE OF TECHNOLOGY UNIVERSITY OF CALIFORNIA MONTANA STATE UNIVERSITY

UNIVERSITY OF NEVADA

NEW MEXICO STATE UNIVERSITY

OREGON STATE UNIVERSITY

UNIVERSITY OF OREGON

OSAKA UNIVERSITY

UNIVERSITY OF SOUTHERN CALIFORNIA
STANFORD UNIVERSITY

UNIVERSITY OF TOKYO

UNIVERSITY OF UTAH

WASHINGTON STATE UNIVERSITY

UNIVERSITY OF WASHINGTON

${ }^{*} \quad{ }^{*} \quad{ }^{*}$
AMERICAN MATHEMATICAL SOCIETY
CHEVRON RESEARCH CORPORATION
TRW SYSTEMS
NAVAL WEAPONS CENTER




\section{Pacific Journal of Mathematics}

\section{Vol. 33, No. $1 \quad$ March, 1970}

Mir Maswood Ali, On some extremal simplexes ................... 1

Silvio Aurora, On normed rings with monotone multiplication........... 15

Silvio Aurora, Normed fields which extend normed rings of integers....... 21

John Kelly Beem, Indefinite Minkowski spaces..................... 29

T. F. Bridgland, Trajectory integrals of set valued functions ........... 43

Robert Jay Buck, A generalized Hausdorff dimension for functions and sets ......................................... 69

Vlastimil B. Dlab, A characterization of perfect rings . . . . . . . . . . . . 79

Edward Richard Fadell, Some examples in fixed point theory ............ 89

Michael Benton Freeman, Tangential Cauchy-Riemann equations and uniform approximation ............................. 101

Barry J. Gardner, Torsion classes and pure subgroups ................ 109

Vinod B. Goyal, Bounds for the solution of a certain class of nonlinear

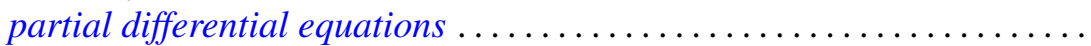

Fu Cheng Hsiang, On C, 1 summability factors of Fourier series at a given

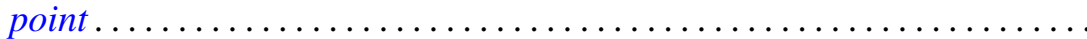

Lawrence Stanislaus Husch, Jr., Homotopy groups of PL-embedding

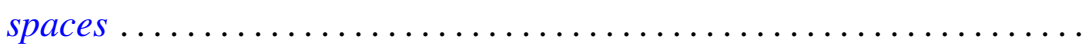

Daniel Ralph Lewis, Integration with respect to vector measures..........

Marion-Josephine Lim, $\mathscr{L}-2$ subspaces of Grassmann product spaces

Stephen J. Pierce, Orthogonal groups of positive definite multilinear functionals

W. J. Pugh and S. M. Shah, On the growth of entire functions of bounded index.

Siddani Bhaskara Rao and Ayyagari Ramachandra Rao, Existence of triconnected graphs with prescribed degrees . . .

Ralph Tyrrell Rockafellar, On the maximal monotonicity of subdifferential

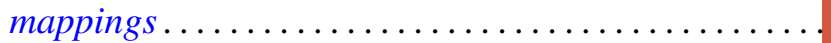

R. Shantaram, Convergence of a sequence of transformations of distribution functions. II ...............................

Julianne Souchek, Rings of analytic functions..............

Ted Joe Suffridge, The principle of subordination applied to functions of several variables...

Wei-lung Ting, On secondary characteristic classes in cobordism

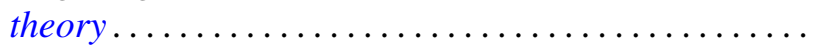

Pak-Ken Wong, Continuous complementors on $B^{*}$-algebras ...

Miyuki Yamada, On a regular semigroup in which the idempotents form a band. 